Abstract 08 Table 1

\begin{tabular}{|c|c|c|c|c|c|c|c|}
\hline & \multirow[t]{2}{*}{$\mathrm{N}$} & \multicolumn{2}{|c|}{ EULAR/ACR 2019 Criteria } & \multicolumn{2}{|c|}{ ACR 1997 Criteria } & \multicolumn{2}{|c|}{ SLICC 2012 Criteria } \\
\hline & & Sensitivity & Specificity & Sensitivity & Specificity & Sensitivity & Specificity \\
\hline & 1,270 & 0.96 & 0.93 & 0.83 & 0.93 & 0.97 & 0.84 \\
\hline $95 \% \mathrm{Cl}$ & & $0.95-0.98$ & $0.91-0.95$ & $0.80-0.85$ & $0.91-0.95$ & $0.95-0.98$ & $0.80-0.87$ \\
\hline \multicolumn{8}{|l|}{ Sex } \\
\hline Women & 1,098 & 0.97 & 0.94 & 0.83 & 0.93 & 0.97 & 0.82 \\
\hline Men & 172 & 0.93 & 0.96 & 0.78 & 0.94 & 0.94 & 0.90 \\
\hline \multicolumn{8}{|l|}{ Ethnicity } \\
\hline Asian & 118 & 0.97 & 0.91 & 0.77 & 0.93 & 0.99 & 0.91 \\
\hline Black & 68 & 0.98 & 1.00 & 0.82 & 1.00 & 0.98 & 0.92 \\
\hline Hispanic & 124 & 1.00 & 0.96 & 0.86 & 0.96 & 1.00 & 0.78 \\
\hline White & 941 & 0.95 & 0.94 & 0.83 & 0.93 & 0.96 & 0.83 \\
\hline \multicolumn{8}{|c|}{ Disease duration } \\
\hline$<1$ year & 34 & 0.89 & 0.92 & 0.56 & 0.92 & 0.89 & 0.92 \\
\hline 1 to $<3 \mathrm{yrs}$ & 196 & 0.97 & 0.96 & 0.81 & 0.95 & 0.98 & 0.88 \\
\hline 3 to $<5$ yrs & 157 & 0.96 & 0.99 & 0.81 & 0.94 & 0.91 & 0.89 \\
\hline$\geq 5$ years & 879 & 0.96 & 0.93 & 0.84 & 0.93 & 0.97 & 0.81 \\
\hline
\end{tabular}

intervals or even higher. Formally, the sensitivity was slightly lower for male patients, corresponding to a higher specificity, but the male $95 \%$ confidence intervals $(0.86-0.98$ for sensitivity, $0.90-0.99$ for specificity) overlapped. While sensitivity appeared independent of disease duration from year 1 on, sensitivity was only $89 \%$ in the first year of disease, identical to the SLICC criteria (89\%) and numerically higher than the ACR criteria (56\%), but all confidence intervals overlapped.

Conclusion While not all subgroups of SLE patients in the validation cohort are of adequate size to fully explore the sensitivity and specificity of the EULAR/ACR 2019 SLE classification criteria in the respective subsets, the point estimates of sensitivity and specificity suggest that the new criteria perform at least reasonably well in all ethnic groups, in men and in early disease. Nevertheless, sensitivity and specificity should be independently validated in larger groups of Asian, Black and Hispanic patients, male patients and in early disease.

\section{REDUCTION OF INTERFERON- $\gamma$ AND ELEVATED BASELINE CYTOTOXIC GENE EXPRESSION IN THE BLOOD ASSOCIATE WITH USTEKINUMAB RESPONSE IN SLE}

${ }^{1}$ Logmane Seridi, ${ }^{1}$ Matteo Cesaroni, ${ }^{1}$ Matt Loza, ${ }^{1}$ Jessica Schreiter, ${ }^{1}$ Kristen Sweet, ${ }^{1}$ Kim Campbell, ${ }^{2}$ Peter Lipsky, ${ }^{3}$ Ronald van Vollenhoven, ${ }^{4}$ Bevra H Hahn, ${ }^{5}$ George C Tsokos,

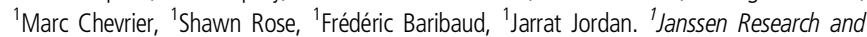
Development, Spring House; ${ }^{2}$ AMPEL BioSolutions, Charlottesville, USA; ${ }^{3}$ Amsterdam Rheumatology and Immunology, Amsterdam, The Netherlands; ${ }^{4}$ UCLA, Los Angeles; ${ }^{5}$ Harvard Medical School, Boston, USA

\subsection{6/lupus-2020-eurolupus.22}

Background/Purpose Ustekinumab (anti-IL-12/23) improved SLE-disease activity vs. placebo in patients with active SLE despite standard therapy. ${ }^{1}$ We investigated whether biomarkers collected in this trial could distinguish responders (UST-R) from non-responders (UST-NR) (response defined by SLE Responder
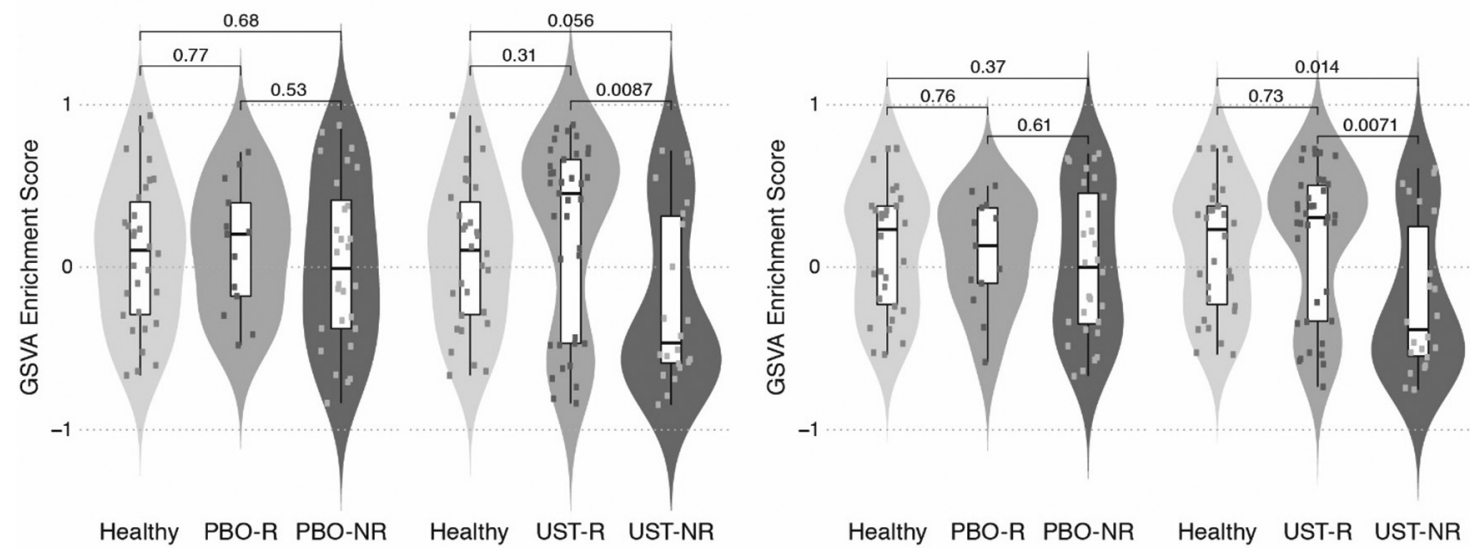

\footnotetext{
1. Seridi L, Cesaroni M, Loza M, et al. Baseline Cytotoxic Gene Expression Associates with Ustekinumab Response in Systemic Lupus Erythematosus [abstract]. Arthritis Rheumatol 2019; 71 (suppl 10). https://acrabstracts.org/abstract/baseline-cytotoxic-gene-expression-associates-with-ustekinumab-response-in-systemiclupus-erythematosus/. Accessed October 17, 2019.

2. Banchereau A, Hong S, Gantarel B, et al. Personalized Immunomonitoring Uncovers Molecular Networks that Stratify Lupus Patients. Cell 2016; 165: 1548-1550
} 
Index-4 at week 24) and if these features could help elucidate the mechanism of action of ustekinumab in SLE.

Methods We examined whole blood RNA and serum at baseline and longitudinally from this trial of 102 seropositive SLE patients, plus age- and sex-matched healthy controls. Targeted proteomic analysis used ELISA, and gene expression analysis used microarray. RNA-Seq was performed on whole blood stimulated in vitro.

Results Changes in serum IL-17A, IL-17F and IL-22 were subtle and did not consistently associate with UST response, while no modulation of type I interferon levels was observed. In contrast, durable reduction in IFN- $\gamma$ protein occurred only in UST-R. These biomarker effects were sustained through Week 48. A non-biased machine-learning algorithm identified a novel 9-gene (PRF1, KLRD1, GZMH, NKG7, GNLY, FGFBP2，TRGC2，TARP，TRGV2) cytotoxic gene-signature (CGS) enriched in baseline blood samples of UST-R vs USTNR, which was corroborated using a published NK-cell CGS (figure 1). No significant differences in these gene-signatures were observed between placebo responders vs non-responders. In contrast, decreased expression levels were observed over the course of 24 weeks for both signatures only in the UST-R population. After whole-blood stimulation with recombinant IL-12, but not IL-23, expression of representative members of the CGS increased.

Conclusion We identified a novel cytotoxic signature in baseline blood samples that associated with UST response in SLE. Targeted biomarker analysis suggests an important role of IL12 blockade in the mechanism of action of UST in SLE.

Acknowledgement This work was supported by Janssen Research \& Development, LLC.

\section{REFERENCE}

1. Van Vollenhoven R.F., Hahn, B.H., Tsokos, G.C., et al. Efficacy and safety of ustekinumab, an IL-12 and IL-23 inhibitor, in patients with active systemic lupus erythematosus: results of a multicentre, double-blind, phase 2, randomised, controlled study. Lancet. 2018;392:1330-1339.

\section{PREDICTION OF RESPONSE TO RITUXIMAB IN SLE USING A VALIDATED TWO-SCORE SYSTEM FOR INTERFERON STATUS}

${ }^{1}$ Adewonuola Alase, 'Zoe Wigston, ${ }^{1}$ Agata Burska, ${ }^{1}$ Antonios Psarras, ${ }^{1}$ Md Yuzaiful Md Yusof, ${ }^{2}$ John Reynolds, ${ }^{3}$ The MASTERPLANS Consortium, 'Miriam Wittman, ${ }^{2}$ Ian N Bruce, ${ }^{1}$ Edward Vital. 'Leeds Institute of Rheumatic and Musculoskeletal Medicine, University of Leeds, Leeds; ${ }^{2}$ Division of Musculoskeletal \& Dermatological Sciences, University of Manchester, Manchester, UK; ${ }^{3}$ The Masterplans Consortium
Background Rituximab (RTX) is used for resistant SLE but clinical response varies. We previously validated two interferon-stimulated gene expression scores (IFN-Score-A and IFN-Score-B) that improved prediction of clinical outcomes in SLE. ${ }^{1}$ IFN-Score-A included most commonly reported ISGs and predicted flares and glucocorticoid requirements. IFN-Score-B included ISGs that respond to multiple IFN subtypes and predicted development of SLE in At-Risk individuals. Diagnosis of SLE was associated with both scores, while only IFN-Score-B was elevated in RA. The British Society for Rheumatology Biologics Registry (BILAG-BR) collects samples for RTX-treated patients in the UK. MASTERPLANS is a consortium to identify predictors of drug response.

Methods Patients were recruited if they were starting a first cycle of RTX for active SLE (BILAG A or 2xBILAG B) despite previous cyclophosphamide or mycophenolate mofetil. Disease activity was measured using BILAG-2004. Clinical response was defined as improvement by $\geq 1$ grade in active BILAG-2004 systems with no worsening in other systems. Whole blood was collected into TEMPUS tubes and RNA extracted. IFN-Scores were measured using a custom Taqman array as previously described [El Sherbiny et al., 2018]. Multivariate logistic regression was used to test IFN-Scores and baseline clinical covariates as predictors of BILAG response at 6 months.

Results Samples were available from 147 patients, of whom 84 had complete baseline and 6 month clinical data available and were included in this analysis. 40/84 (47.6\%) patients had BILAG response at 6 months. In univariate and multivariate analysis, high IFN-Score-B expression was significantly associated with clinical response (see table 1 ).

Conclusions This preliminary analysis suggests that assessment of IFN activity has a role in predicting response to RTX. A novel IFN score (Score B) was more predictive than classic ISGs (Score A). These results add to a body of work showing that IFN-Score-B predicts clinically significant outcomes independently of overall IFN activity. Future work will analyse this biomarker in a larger cohort of patients and integrate with other putative clinical and biological predictors of response.

Acknowledgements This project was funded by a grant from the Medical Research Council for MASTERPLANS.

\section{REFERENCE}

1. El-Sherbiny YM, Vital EM. Sci. Rep. 2018;8:5793.

\begin{tabular}{|c|c|c|c|c|c|c|}
\hline Predictor & $\begin{array}{l}\text { Non-responders } \\
(n=44)\end{array}$ & Responders $(n=40)$ & $\begin{array}{l}\text { Univariable OR } \\
(95 \% \mathrm{Cl})\end{array}$ & $\mathbf{P}$ & $\begin{array}{l}\text { Multivariable OR } \\
(95 \% \mathrm{Cl})\end{array}$ & $\mathbf{P}$ \\
\hline Age (mean, 95\% Cl) & $40.5(36.2,44.8)$ & $40.9(36.2,45.6)$ & $1.01(0.99,1.03)$ & 0.18 & $0.99(0.96,1.03)$ & 0.76 \\
\hline \multicolumn{7}{|l|}{ Organs affected } \\
\hline Mucocutaneous & $22 / 44$ & $23 / 40$ & $0.86(0.46,1.60)$ & 0.64 & $1.02(0.38,2.75)$ & 0.96 \\
\hline Musculoskeletal & $21 / 44$ & $18 / 40$ & $0.72(0.39,1.35)$ & 0.72 & $0.42(0.15,1.24)$ & 0.11 \\
\hline Renal & $21 / 44$ & $16 / 40$ & $0.86(0.47,1.60)$ & 0.65 & $0.29(0.09,0.97)$ & 0.04 \\
\hline Cardiovascular & 9/44 & $5 / 40$ & $1.25(0.55,2.82)$ & 0.59 & $0.63(0.16,2.39)$ & 0.49 \\
\hline Neurological & $7 / 44$ & $6 / 40$ & $0.85(0.35,2.06)$ & 0.73 & $0.77(0.17,3.47)$ & 0.73 \\
\hline IFN-Score-A (per unit) & $2.49(1.77,3.19)$ & $1.74(1.10,2.39)$ & $0.84(0.68,1.04)$ & 0.12 & $1.60(0.93,2.74)$ & 0.08 \\
\hline IFN-Score-B (per unit) & $2.36(1.98,2.73)$ & $1.76(1.43,2.09)$ & $0.60(0.39,0.93)$ & 0.02 & $0.27(0.09,0.76)$ & 0.01 \\
\hline
\end{tabular}

